\title{
Supporting clinical research in the NHS in England: the National Institute for Health Research Dementias and Neurodegenerative Diseases Research Network
}

\author{
Piers Kotting*1, Kris Beicher², lan G McKeith² and Martin N Rossor ${ }^{1}$
}

\begin{abstract}
Clinical research is best done when aligned with clinical care - that is, when the patient can be identified, recruited and, in many instances, researched in parallel with the delivery of clinical service. However, to achieve this effectively requires identification of the additional cost to the National Health Service clinical support services and the development of an appropriately skilled workforce. The National Institute for Health Research (NIHR) Cancer Research Network demonstrated the value of dedicated research support in terms of the number of patients recruited into clinical trials. Building on this model, the NIHR in England funded the Dementias and Neurodegenerative Diseases Research Network (DeNDRoN). DeNDRoN is now in its sixth year and has established a geographically widespread network of research support staff and research leadership managed by a central coordinating centre. Success can already be measured by a significant increase in the number of patients entering studies and the speed with which both commercial and noncommercial studies are completed. There are also early indications that the network will result in improved patient outcomes.
\end{abstract}

\section{DeNDRoN: background, context and purpose}

The Dementias and Neurodegenerative Diseases Research Network (DeNDRoN) was established in 2005 along with research networks for diabetes, medicines for children and for stroke. Together with existing research networks

*Correspondence: p.kotting@ucl.ac.uk

'Department of Neurodegenerative Diseases, Institute of Neurology, University College London, London WC1N 3BG, UK

Full list of author information is available at the end of the article for cancer and for mental health, these became the six topic-specific clinical research networks in England. In 2006 a comprehensive and radical government strategy for health research in England was published [1], leading to the establishment of two further research networks: a Comprehensive Clinical Research Network and a Primary Care Research Network. All eight English clinical research networks were brought together as part of the new National Institute for Health Research (NIHR). The NIHR is a publicly funded body in England, the purpose of which is to create a health research system in which the National Health Service (NHS) supports outstanding individuals, working in world-class facilities, conducting research focused on the needs of patients and the public. The NIHR manages its activities through four main work strands, as shown in Figure 1. The NIHR Clinical Research Network structure is shown in Figure 2.

During 2007 and 2008 approximately $£ 500$ million (\$780 million) of research support funding was withdrawn from NHS trusts in England and replaced with new mechanisms to support the delivery of clinical research (see Box 1). The NIHR Clinical Research Network is the largest part of this new research infrastructure in England.

As part of the NIHR Clinical Research Network, DeNDRoN has five key aims: to ensure that patients and healthcare professionals from all parts of England are able to participate in and benefit from clinical research in dementia, Parkinson's disease, motor neurone disease, Huntington's disease and other neurodegenerative diseases; to integrate health research and patient care in these conditions; to improve the quality, speed and coordination of clinical research in these conditions; to streamline and performance-manage NHS support (see Box 1) for eligible studies [2] (see Box 2) to ensure that the NHS Support Costs are met in a timely and efficient manner; and to strengthen research collaboration with life-sciences industries and ensure that the NHS can meet the health research needs of industry. 


\section{National Institute for Health Research}

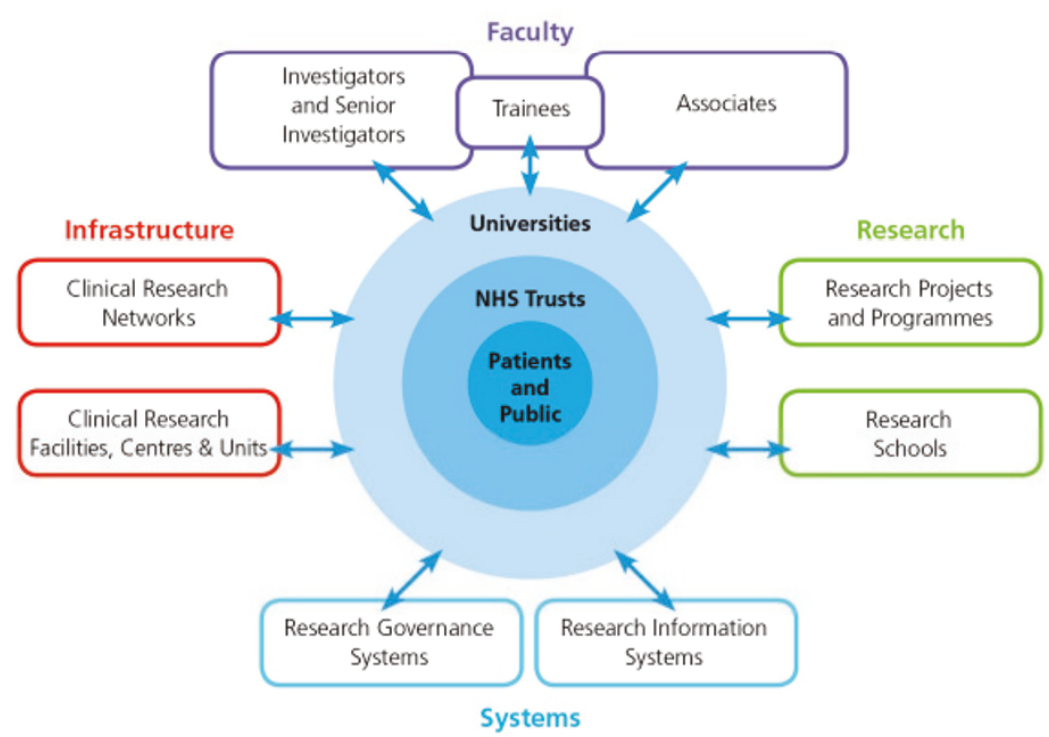

Figure 1. National Institute for Health Research in England. NHS, National Health Service.

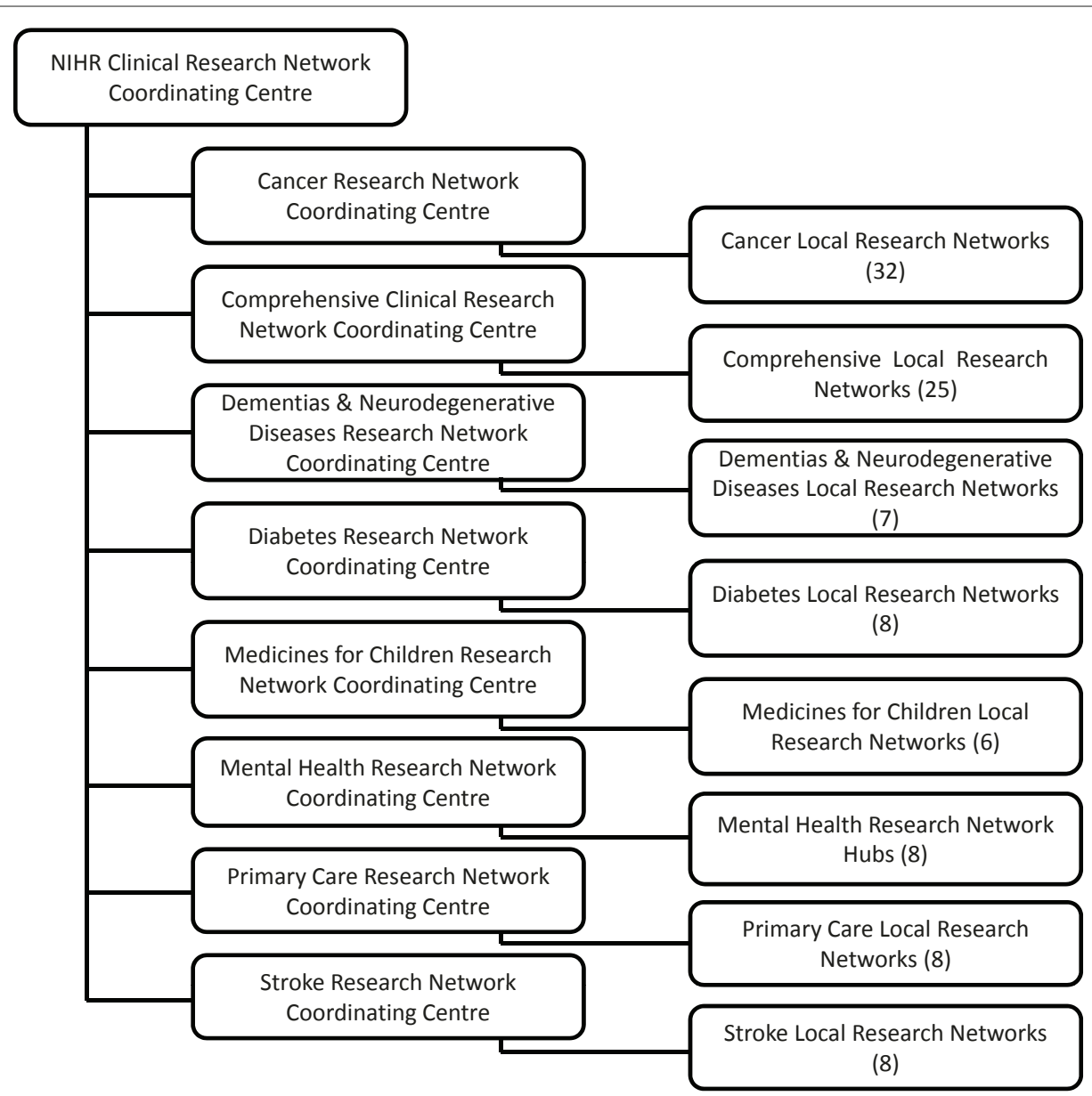

Figure 2. Structure of the National Institute for Health Research Clinical Research Network. NIHR, National Institute for Health Research. 


\section{Box 1. How clinical research is funded in England \\ Clinical research studies comprise a number of component activities, which, for the purpose of agreeing funding arrangements, are attributed to one of three broad cost categories: Research Costs are the costs of the R\&D itself that end when the research ends. They relate to activities that are being undertaken to answer the research questions. These costs are met by research funding bodies such as the Medical Research Council or Alzheimer's Research UK. \\ NHS Treatment Costs are the patient care costs, which would continue to be incurred if the patient care service in question continued to be provided after the R\&D study had stopped. These costs are met by the NHS through its clinical commissioning systems. \\ NHS Support Costs are the additional patient care costs associated with the research, which would end once the R\&D study in question had stopped, even if the patient care involved continued to be provided. Until 2006 these costs were funded by the government directly to NHS trusts under the Culyer system. Following a period of transition, since 2008 the NHS support costs have been met through the NIHR infrastructure.}

In addition to Clinical Research Network-wide aims, DeNDRoN has two further goals. Despite the scale of the social, health and economic burden of dementia and neurodegenerative diseases, there has historically been relatively little investment in research into these conditions in the UK compared with other diseases [3-5]. Notwithstanding this, there are a number of internationally recognised centres of excellence in dementia and neurodegenerative diseases research, and the UK ranks second in the world on both research output and citation impact in dementia research [5]. DeNDRoN aims to build on this existing expertise to increase the dementia and neurodegenerative diseases clinical research leadership capability in England.

The clinical service pathways for people with dementias and neurodegenerative diseases in England are highly complex. People are diagnosed and treated across a combination of primary and secondary NHS settings, and by a number of specialists including old-age psychiatrists, neurologists and geriatricians. These specialists have different training paths, professional bodies and research traditions, and operate in different NHS settings, leading to relatively little collaboration between specialties. DeNDRoN aims to increase collaboration between these specialties to develop and deliver dementia and neurodegenerative diseases research.

\section{DeNDRoN: increasing the NHS leadership and workforce capacity to deliver dementia research in England}

National coordination of the network

Following an open, peer-reviewed competition, a contract was awarded in September 2005 by the Department

\section{Box 2. Eligibility for inclusion in the NIHR Clinical Research Network Portfolio}

For a research study to qualify for receiving NHS Support Costs (see Box 1) from the NIHR Clinical Research Network it must meet certain criteria. In summary, studies must be of clear value to the $\mathrm{NHS}$ and must have full research funding that has been awarded as a result of open competition across England with high-quality peer review.

Studies funded by the NIHR, other areas of central government, and NIHR noncommercial partners are automatically eligible for the portfolio. NIHR noncommercial partners are organisations that meet predefined conditions in their funding processes. Other studies - such as commercial contract research, investigator-initiated, commercial-collaborative studies or academic studies funded by overseas governments or charities require formal consideration via an adoption process. The NIHR Clinical Research Network manages the adoption process for both commercial and noncommercial studies on behalf of the Department of Health.

of Health in England to the Institute of Neurology at University College London (UCL) to run DeNDRoN in collaboration with Newcastle University. A national Coordinating Centre was established by collaborators from UCL, Newcastle University, Manchester University, Sheffield University and Cardiff University. The contract was extended for a further 5 years in 2010 following successful international peer review. The Coordinating Centre is led by Directors Martin Rossor at UCL and Ian McKeith at Newcastle University, supported by Associate Directors David Burn at Newcastle University (lead for Parkinson's disease), Steve Iliffe at UCL (primary care, and patient and public involvement), John O'Brien at Newcastle University (dementia), Anne Rosser at Cardiff University (Huntington's disease) and Pamela Shaw at Sheffield University (motor neurone disease).

The DeNDRoN Coordinating Centre provides leadership to the network and manages it on behalf of the NIHR. It is responsible for developing and quality assuring local and national infrastructure, systems and processes to meet the aims of the network. It coordinates network participation in NIHR Clinical Research Network portfolio studies and promotes the active involvement of patients and the public in research.

The structure of the Coordinating Centre is illustrated in Figure 3. The core business of the supporting portfolio development and delivery is managed by an operations team based in Newcastle, Sheffield and Cardiff. A programme office at UCL supports the delivery of projects to address areas of strategic national importance. Finance, patient and public involvement and communications functions are also provided from UCL. The Coordinating Centre employs 13.8 whole-time-equivalent staff and 2.3 whole-time-equivalent directors. 


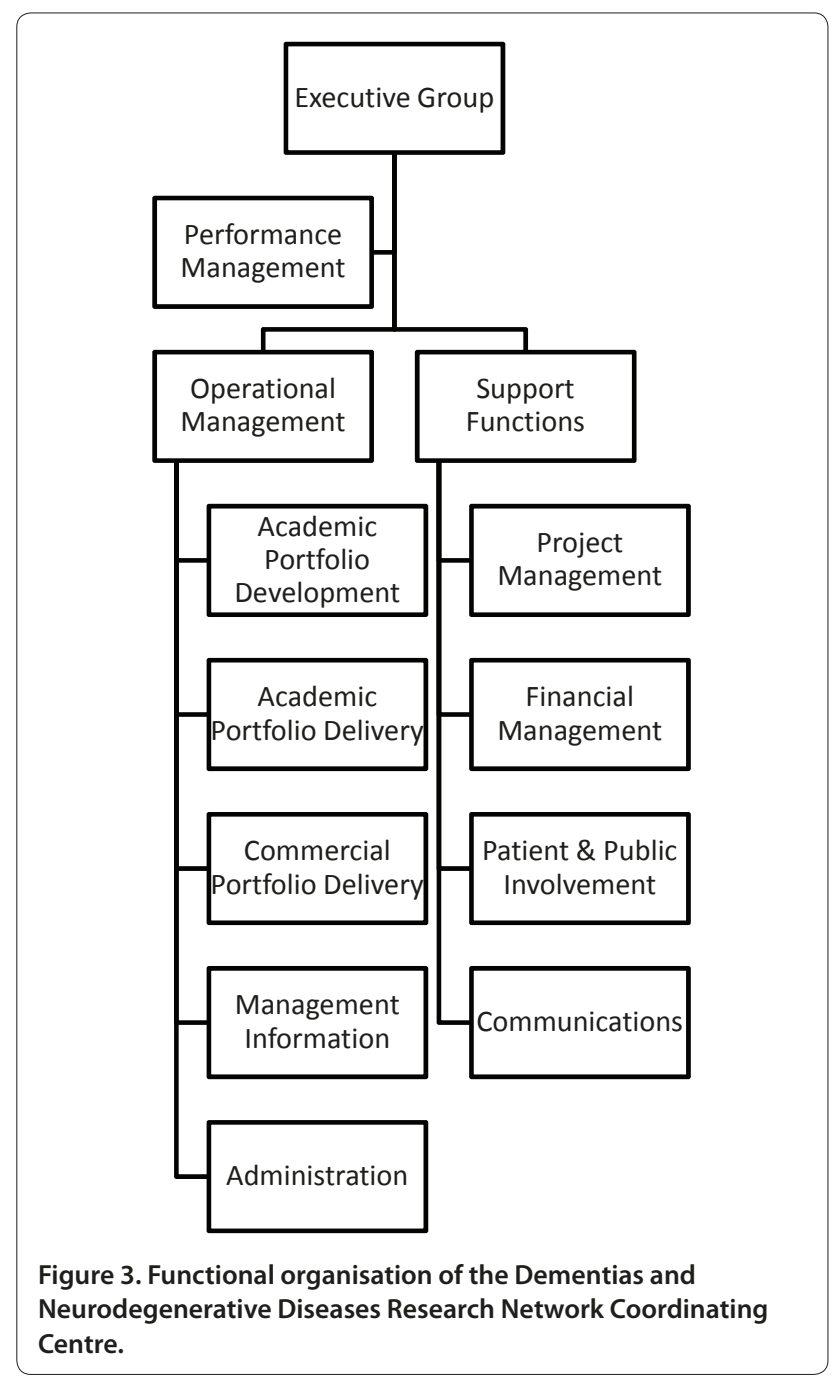

\section{Local research infrastructure}

Unlike the Cancer Research Network that preceded them or the Comprehensive Clinical Research Network and Primary Care Research Network that followed, the other topic-specific clinical research networks were established with sufficient funding to develop local infrastructure in selected parts of England. Following an open, national competition, DeNDRoN therefore established seven Local Research Networks (LRNs) in mid-2006 covering $50 \%$ of the population of England. Additional resources were invested in a further three regions to support research in motor neurone disease and Huntington's disease due to their lower prevalence (see Figure 4).

Each of the seven DeNDRoN LRNs is hosted by an NHS trust, either an acute hospital or mental health trust, which manages the approximately $£ 500,000(\$ 780,000)$ government funding for local NHS infrastructure to support relevant clinical research. The core operational activities of each of the DeNDRoN LRNs are: supporting

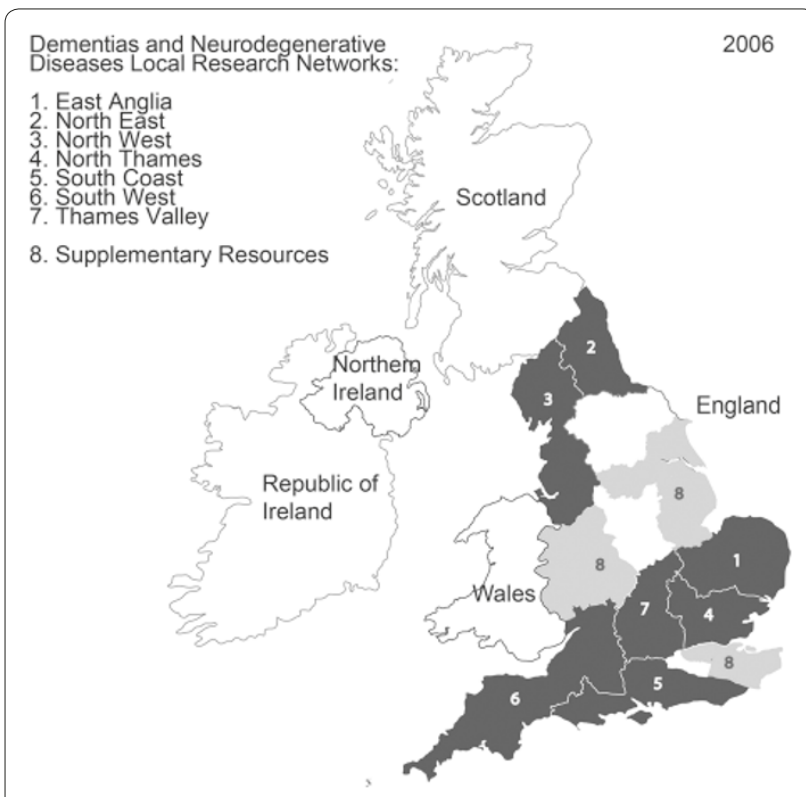

Figure 4. DeNDRoN Local Research Network regions, 2006

site selection and feasibility assessment for future studies; supporting local research management and governance processes and facilitating study set-up; recruiting patients to participate in portfolio studies (see Box 2 and the 'DeNDRoN research portfolio' section below); managing the progress of patients through the research process; carrying out investigations, assessments and tests attributed as NHS Support activities (see Box 1); collecting, collating and submitting research performance management data; and promoting the active involvement of patients and the public in research. In addition, the LRNs undertake a number of activities to support the development of the network, such as: delivering service improvement projects; raising awareness of research in patient, carer and clinical communities; and workforce development and training, including developing new investigators.

Under the overall guidance of the DeNDRoN Coordinating Centre, LRNs have invested their resources according to local requirements, leading to variability. Factors influencing the development of LRN infrastructure include local research interests and expertise, existing research infrastructure and the clinical pathways in the local NHS. The LRNs can be characterised by the relative centralisation or decentralisation of their resources. For example, in the North Thames region where existing research activity and expertise was focused in central London and transport links are good, research support staff were employed in the host trust and travelled to outlying locations as required. In the South West, the largest geographical region, resources were invested in 
two hubs from which support was offered to neighbouring sites. In the South Coast region, where there are a number of sites with equivalent populations spread across the region, staff were employed and worked locally at each site. As the network has developed and the number of sites actively recruiting patients has increased, each of the LRNs has evolved and adapted its resourcing model. Today the networks increasingly run mixed models with some centralised staff and some decentralised staff, with the general trend towards decentralisation. This new infrastructure represents a significant move towards integration of clinical care and research compared with the largely university-based research teams that delivered dementia research prior to DeNDRoN.

The development of local network infrastructure was slow in the first year while contracts were negotiated and key management staff employed. During the second year the numbers of staff grew rapidly, but this period was also characterised by high staff turnover at almost $20 \%$. Turnover remained high compared with the average NHS turnover rate of $13 \%$ [6] during this period. Evidence from exit interviews suggests three main reasons for this: a lack of understanding or clarity about job descriptions and roles; insufficient research activity (see the 'Portfolio growth' section below); and lack of workforce stability itself. Since April 2009, with increasing understanding of the role requirements and sufficient portfolio activity, the workforce has become stable (turnover $<10 \%$ ) and increasingly experienced and sophisticated.

The network aims to maximise the amount it invests in directly supporting the delivery of clinical research studies. As the workforce has grown, the proportion of total DeNDRoN expenditure on frontline staff grew from $27 \%$ in $2006 / 07$, to $57 \%$ in $2007 / 08$, to $68 \%$ in $2008 / 09$, at which level it has remained. The majority of LRN infrastructure (67\%; see Table 1) is invested in research support staff, mainly research nurses as well as those from allied health professions such as psychologists and occupational therapists. The next largest investment is in research administration and data management (17\%), followed by supporting the time of clinical researchers, either in delivering research or in providing local leadership (8\%), and management of the networks (8\%).

The activities of staff vary considerably by region and depending on the particular demands of the local portfolios at any given time. However, an analysis of the overall allocation of time to different activities across the network shows that a broad range of activities are supported (see Table 2), with research governance and study administration accounting for $19 \%$ of time, patient recruitment for $27 \%$, data collection and submission for $28 \%$, and $26 \%$ of time dedicated to development and running of the networks.
Table 1. Total staffing establishment of DeNDRoN Local Research Networks, 2011

\begin{tabular}{lccc}
\hline & $\begin{array}{c}\text { Number } \\
\text { of posts }\end{array}$ & $\begin{array}{c}\text { Whole-time } \\
\text { equivalence }\end{array}$ & $\begin{array}{c}\text { Percentage of } \\
\text { whole-time } \\
\text { equivalent }\end{array}$ \\
\hline Clinical research leads & 38 & 3.6 & 3 \\
Senior doctors & 14 & 4.3 & 4 \\
Junior doctors & 2 & 0.7 & 1 \\
Research support staff & 114 & 72.6 & 67 \\
Management staff & 10 & 8.7 & 8 \\
Data managers & 3 & 3 & 3 \\
Administrative staff & 21 & 15.2 & 14 \\
Total & 202 & 108.1 & \\
\hline
\end{tabular}

DeNDRoN, Dementias and Neurodegenerative Diseases Research Network.

Supporting dementia research in the remainder of England Prior to the establishment of DeNDRoN, an NIHR Mental Health Research Network (MHRN), led from King's College London and Manchester University, had been established in 2003. As many of the clinical services for dementia and mental health are provided by the same NHS trusts, the MHRN has also been able to support dementia research. In particular, the MHRN has supported major multicentre trials in regions without DeNDRoN LRNs - which has meant that the active dementia research centres in the East and West Midlands and in south London have received equivalent network support.

In 2007 the NIHR Comprehensive Clinical Research Network was established across the whole of England, to provide network support to the areas of health research without topic-specific networks, and to provide infrastructure for the topics in areas of the country without topic LRNs. In 2009 funding was made available by the DeNDRoN LRNs through the Coverage Project to support pump-priming of network posts in the non-LRN regions, with a view to the Comprehensive Local Research Networks (CLRNs) continuing to fund these posts as they matured. In 2011 the CLRNs and DeNDRoN collaborated on one of the projects (Integrating Networks and Embedding Research in Clinical Practice (INTERACT)) initiated by the Ministerial Advisory Group on Dementia Research [7], through which dedicated dementia leadership and portfolio management processes have been put in place in all nonDeNDRoN LRN regions of England (see Figure 5). With these new arrangements there are dedicated research leaders for dementia, Parkinson's disease, motor neurone disease and Huntington's disease in 11 regions of England, who are networked through the national DeNDRoN Portfolio Management Groups for each disease. This represents a significant increase in the 
Table 2. Percentage of front-line staff time spent on different activities, 2008

\begin{tabular}{lc}
\hline Activity & Staff time (\%) \\
\hline Data collection & 18 \\
Pre-screening & 16 \\
Screening and consenting & 11 \\
Completing case report forms & 10 \\
Research governance & 10 \\
Training and development & 10 \\
General administration & 10 \\
Study administration & 9 \\
Staff supervision & 3 \\
Patient and public involvement & 3 \\
\hline
\end{tabular}

dedicated and funded leadership capacity for dementia and neurodegenerative disease clinical research in England. During 2012 INTERACT will be delivering 20 local service improvement projects aimed at further integrating research into core dementia care pathways in the NHS.

\section{International collaboration}

Much of DeNDRoN's focus has been and remains on improving the capacity and performance of clinical research performance in the UK. To date there has been little international collaboration with other similar networks, except through delivery of international studies. The exception to this is the UK Huntington's Disease Network, part of DeNDRoN, which plays a major role in the European and global Huntington's disease networks.

\section{DeNDRoN: supporting the development of dementia research in England}

DeNDRoN is predominantly a delivery organisation, providing the infrastructure described above to support the delivery of clinical research studies in the NHS. However, DeNDRoN also has a mandate to support the development of its academic research portfolio to increase the quantity and quality of dementia and neurodegenerative diseases research in England, which it does through development activities focused in three main areas.

The first area is to support collaboration between researchers, clinicians, patients and carers in the identification of new research questions and the writing of grant applications for submission to research funding bodies. The DeNDRoN Coordinating Centre funds and supports activities at different stage of the protocol and grant application process, as described in Table 3. These portfolio development activities are led by the national Clinical Studies Groups.

The second area is to improve the feasibility and deliverability of studies. The network has supported the

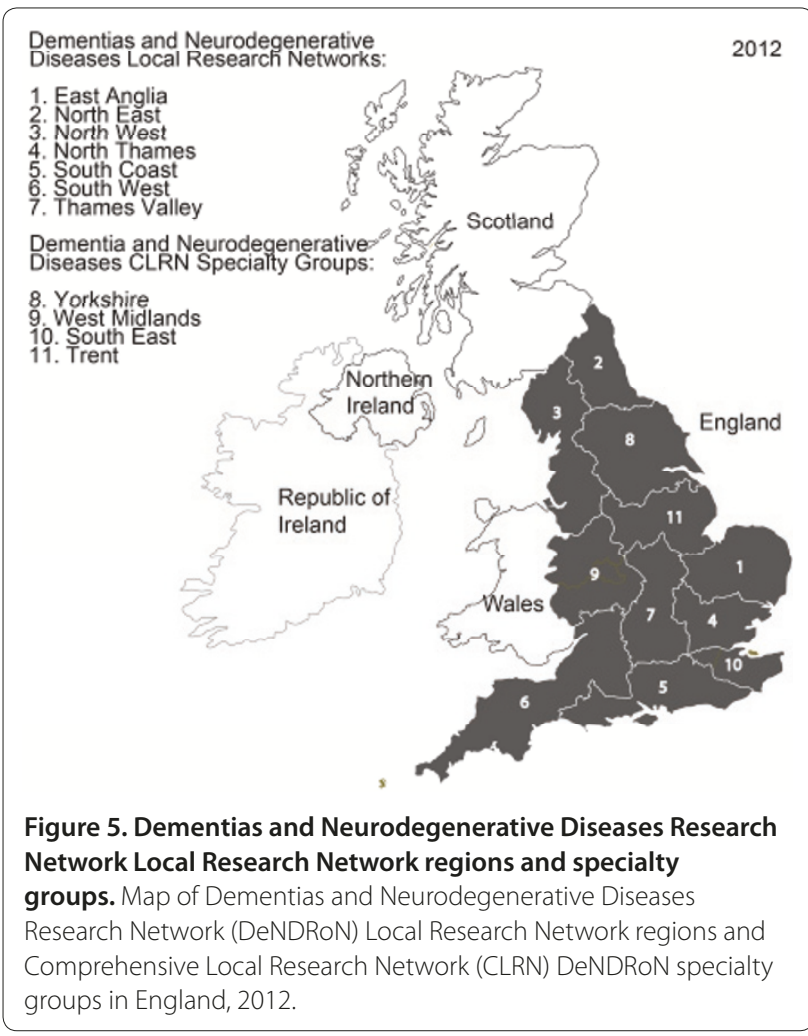

delivery of more than 130 dementia studies since 2006. Increasingly sophisticated processes are being developed to enable researchers to draw on data and experience from this activity to support them in developing feasible protocols - in particular, drawing on the knowledge of LRN staff responsible for delivery.

The final area is to support active patient and public involvement in protocol development. DeNDRoN provides researchers with two levels of support with involving patients and carers in protocol development. Guidance and advice is provided via an online toolkit and in person by the DeNDRoN Patient and Public Involvement Coordinator. A managed service is provided for DeNDRoN Writing Groups, through which DeNDRoN provides: expert advice in developing a patient and public involvement plan to support the application; administrative support to arrange specific patient and public involvement activities; management support with conducting specific patient and public involvement activities; and funding to cover the costs of coordination of specific patient and public involvement activities.

\section{DeNDRoN research portfolio}

A common misconception about the NIHR Clinical Research Network is that it only supports multicentre randomised interventional studies. In fact, the DeNDRoN portfolio comprises predominantly nonrandomised 
Table 3. DeNDRoN academic portfolio activities

\begin{tabular}{|c|c|c|c|}
\hline & What are they? & How were/are they established? & Current groups \\
\hline \multirow[t]{5}{*}{ Clinical Studies Groups } & \multirow{5}{*}{$\begin{array}{l}\text { Ongoing national multidisciplinary } \\
\text { groups undertaking horizon scanning, } \\
\text { idea generation, prioritisation, and so } \\
\text { forth, in a disease area }\end{array}$} & \multirow{5}{*}{$\begin{array}{l}\text { Through open national competition } \\
\text { during 2006. Annual open calls for } \\
\text { new membership. Approximately } 100 \\
\text { members in total }\end{array}$} & Dementias \\
\hline & & & Parkinson's disease \\
\hline & & & Motor neurone disease \\
\hline & & & Huntington's disease \\
\hline & & & Primary care \\
\hline \multirow[t]{3}{*}{ Task Forces } & \multirow{3}{*}{$\begin{array}{l}\text { Time-limited multidisciplinary groups } \\
\text { focused on idea generation in specific } \\
\text { topic area(s) }\end{array}$} & \multirow{3}{*}{$\begin{array}{l}\text { Topics identified through CSGs or in } \\
\text { discussion with partner organisations. } \\
\text { Run in collaboration with specific } \\
\text { partners }\end{array}$} & $\begin{array}{l}\text { Vascular dementia (with Stroke Association and } \\
\text { Alzheimer's Society) }\end{array}$ \\
\hline & & & End-of-life care (with Alzheimer's Society) \\
\hline & & & Assistive technology (with Alzheimer's Society) \\
\hline Writing Groups & $\begin{array}{l}\text { Group of research collaborators working } \\
\text { together to submit a multicentre grant } \\
\text { application to an NIHR-eligible funding } \\
\text { body }\end{array}$ & $\begin{array}{l}\text { Through CSGs; as outputs from } \\
\text { task forces; through open calls for } \\
\text { expressions of interest; or in response to } \\
\text { application from researchers }\end{array}$ & $\begin{array}{l}25 \text { groups were supported in } 2011 . \\
\text { Approximately one-half have already submitted } \\
\text { grant applications }\end{array}$ \\
\hline
\end{tabular}

CSG, Clinical Studies Group; DeNDRoN, Dementias and Neurodegenerative Diseases Research Network; NIHR, National Institute for Health Research.

observational studies - and 36\% of all studies are single centre (see Figure 6), representing the type of dementia research currently undertaken in England. The relative proportion of studies by disease area is shown in Figure 7 . DeNDRoN supports a wide range of trial designs, including early and late-phase randomised control trials of new interventions and agents (34\%; for example, immunotherapy in Alzheimer's disease), identifying genetic risk factors for specific disorders (6\%; for example, the Motor Neurone Disease Association DNA Bank) and observational studies (56\%; for example, patient and carer outcomes, health economic factors and care pathways).

Widening the scope from dementia to neurodegenerative diseases allows greater interdisciplinarity and reflects the way that the research community is beginning to organise itself. Huntington's disease is a prototypic dementia and the other neurodegenerative diseases all share cognitive impairment to a variable extent. There is now greater engagement across neurology, psychiatry and geriatric medicine than any single disease network could achieve. This does generate specialism and service delivery boundary issues, but tackling these is important for research as they reflect service delivery issues in the clinical setting.

\section{Impact of DeNDRoN Portfolio growth}

The increased collaboration across the dementia research community and between specialties can be seen both in terms of the growth of the academic dementia portfolio and the growth in participation of patients and clinicians. The number of dementia studies on the DeNDRoN portfolio open to recruitment has grown from 16 in $2006 / 07$ to 64 in $2010 / 11$ (see Figure 8 ). The growth in the number of academic studies between 2006/07 and
2008/09 reflects the identification of eligible studies and their addition to the portfolio, rather than an actual growth in the research activity. Since 2008/09, when close to $100 \%$ of eligible studies were included in the portfolio, any growth describes a real increase in activity. The number of academic dementia studies recruiting in 2008/09 and 2009/10 was the same (40 studies). There was a $17 \%$ increase in 2010/11, with 47 academic studies recruiting; and there was an increase in government funding of dementia research from $£ 12.7$ million in $2009 / 10$ to $£ 18.6$ million in 2010/11 [8]. Another 15\% increase is expected for 2011/12, with 43 academic studies starting recruitment in the first 6 months and a further 11 expected to open within the year.

During 2011 the NIHR issued its first ever Themed Call for Dementia Research across most of its clinical research funding programmes. DeNDRoN supported the community to respond to the call through its Clinical Studies Groups, Writing Groups and more generally through providing input into deliverability and patient and public involvement. Approximately $£ 17$ million will be committed to 18 new research projects through the call, exceeding original expectations [9]. DeNDRoN appears to have been effective in supporting the development of more successful applications than might have occurred without network involvement. However, as the network matures and with the increase in funding for dementia research announced in March 2012 [9], could DeNDRoN support even greater output from existing researchers by taking a more strategic role in portfolio development? This is primarily a question for researchers and research funders.

\section{Participation in research}

The DeNDRoN infrastructure has had a major impact on increasing participation within the LRN regions. In 2007, 

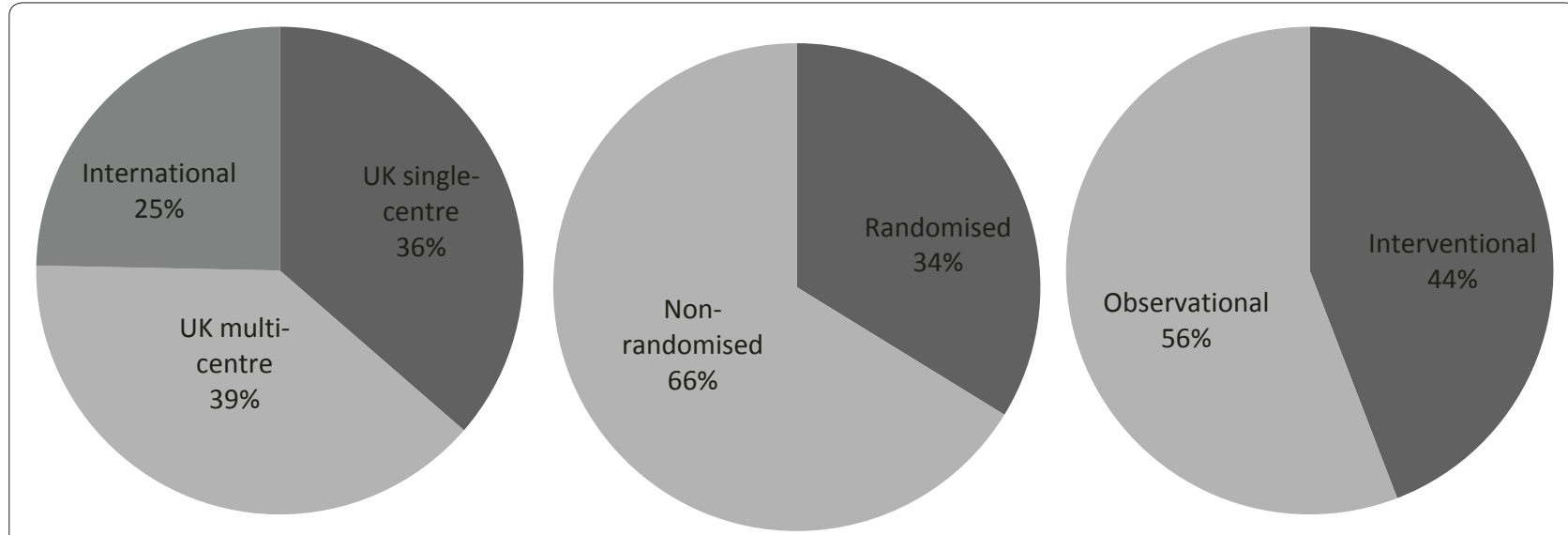

Figure 6. Balance of the Dementias and Neurodegenerative Diseases Research Network dementia portfolio, 2011. Number of studies $=66$.

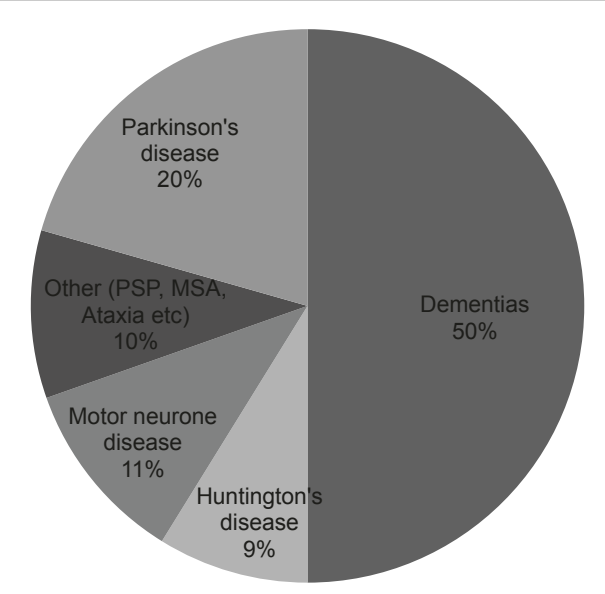

Figure 7. Balance of the Dementias and Neurodegenerative Diseases Research Network portfolio by disease, 2011. Total number of studies on portfolio = 132. MSA, multiple system atrophy; PSP, progressive supranuclear palsy.

there were 31 NHS sites actively involved in 25 NIHR portfolio dementia studies within DeNDRoN LRN regions; in 2010/11, 188 sites participated in 56 studies. During this period the number of patients entering studies rose from 911 to 6,700. Through collaboration with the CLRN, capacity and participation in non-LRN areas is now also increasing with 191 sites supporting 25 portfolio dementia studies in 2010/11 and recruiting 3,950 patients (see Figures 8 and 9).

\section{Performance of studies}

In addition to increasing NHS capacity to deliver dementia research, DeNDRoN has also improved the performance of individual studies. In the 24 months to 31 December 2010, the average time taken to recruit first subject into academic portfolio studies was 126 days with a median of 50 days. Since the introduction of a dedicated
Portfolio Delivery Manager and Portfolio Management Groups in January 2011, the average is 91 days with a median of 29 days. Although reliable baseline data are not available about delivery of studies on time to target, it is estimated that the proportion of academic and commercial studies completing on target within predefined timelines in 2006 was $30 \%$ and $40 \%$, respectively, which is in line with published data [10]. In the current year $(2011 / 12)$ this proportion is $73 \%(n=15)$ and $87 \%(n=8)$, respectively. Comparative performance between regions with and without DeNDRoN LRNs cannot be assessed for academic dementia studies as multicentre studies have received equivalent support from the MHRN in non-DeNDRoN LRN regions. In the Parkinson's disease portfolio where there has been no alternative support outside DeNDRoN LRNs until the CLRN infrastructure developed, sites in DeNDRoN LRN regions have been shown to recruit participants twice as quickly as those outside $[11,12]$.

The impact of the DeNDRoN LRN infrastructure on the capacity and performance of NHS sites in commercial dementia research can be demonstrated in comparison with non-LRN areas that had no Clinical Research Network support prior to the CLRN and in which the MHRN that did not support commercial dementia studies (see Figure 10). The rate of growth (2007/08 to 2010/11) in the number of studies is the same in both comparison groups, reflecting the underlying increase in industry-sponsored studies coming to the UK. However, the rate of growth of study sites in DeNDRoN regions is almost double that outside DeNDRoN. Although commercial research sponsors still display a preference for using sites with whom they have worked before, DeNDRoN has been successful at introducing new sites and proving that these can deliver commercial trials. The approach involved has largely been to introduce new investigators as satellite sites to an experienced 


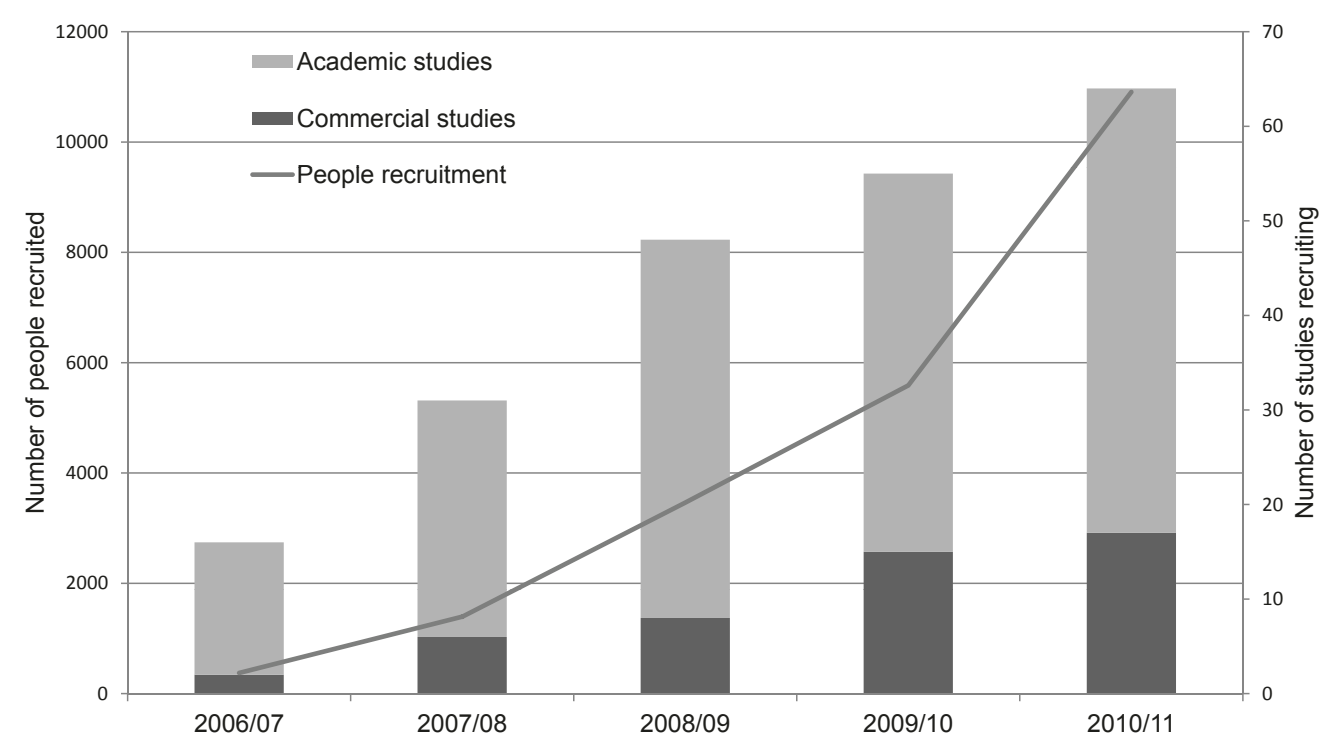

Figure 8. Total Dementias and Neurodegenerative Diseases Research Network dementia portfolio activity, 2006 to 2011.

investigator in less complex studies, to develop their skills prior to taking on more complex interventional trials. The work entailed in developing new investigators and securing commercial trials for them should not be underestimated, but is critical in order to increase the capacity of the UK to deliver commercial research.

Of perhaps more interest in assessing the network model is the fact that the rate of growth of recruitment in DeNDRoN regions is 13 times higher than that outside the network. One of the key benefits for commercial sponsors working with the network is the additional coordination of the NHS sites participating in their studies. The impact of this is an increased ability to share learning across sites and intervene to ensure successful delivery. The LRNs also provide a flexible workforce that can be deployed as required to support peaks in activity, provide cover for absent staff or even open new sites if required. In the most recently completed commercially sponsored study, all 16 DeNDRoN LRN sites completed on target within the agreed timeframe, compared with three out of eight sites outside DeNDRoN LRNs. Of the DeNDRoN sites, 12 of the 16 had become active in commercial research with the support of the network - for six of these sites, this was their first commercial study.

\section{Registers of interest in research}

In addition to increasing capacity, the network is also identifying, piloting and implementing new ways of delivering clinical research in the NHS. For example, DeNDRoN has been piloting the use of research registers - lists of people with dementia who have given their consent to be contacted about future research activity - across a number of its LRNs.

Initial findings from the North Thames Dementia Register (DemReg) pilot suggest that using these lists greatly increases rates of recruitment and reduces the costs $[13,14]$. During the first 6 months of the EVIDEM-E project, recruitment was via the clinical services of the local NHS Mental Health Trust [15]. One person was recruited to the study for every 75 people screened and sent information packs $(n=300)$. Subsequently the study started recruiting by writing to people who had already registered their interest in studies through DemReg. This approach more than doubled recruitment rates, with one person recruited per 29 people screened $(n=88)$. When the research team contacted people on DemReg in person (either by telephone or face to face in the clinic), the recruitment rate was one person per two people screened $(n=30)$. In addition to the increase in recruitment rates, the use of DemReg significantly reduced the resources required in the research team to recruit patients. During the original recruitment phase, 130 hours of research team time were spent explaining the trial to the clinical teams, compared with 3 hours working with the DemReg coordinators to run the relevant queries.

In 2012 DeNDRoN will be leading another of the projects of the Ministerial Advisory Group on Dementia Research (Recruitment and Feasibility Tools Project), assisting with the development and use of registers to support dementia research across England, and developing a nationally consistent system to support participation in research as announced by the Prime Minister in his challenge on dementia [9]. 


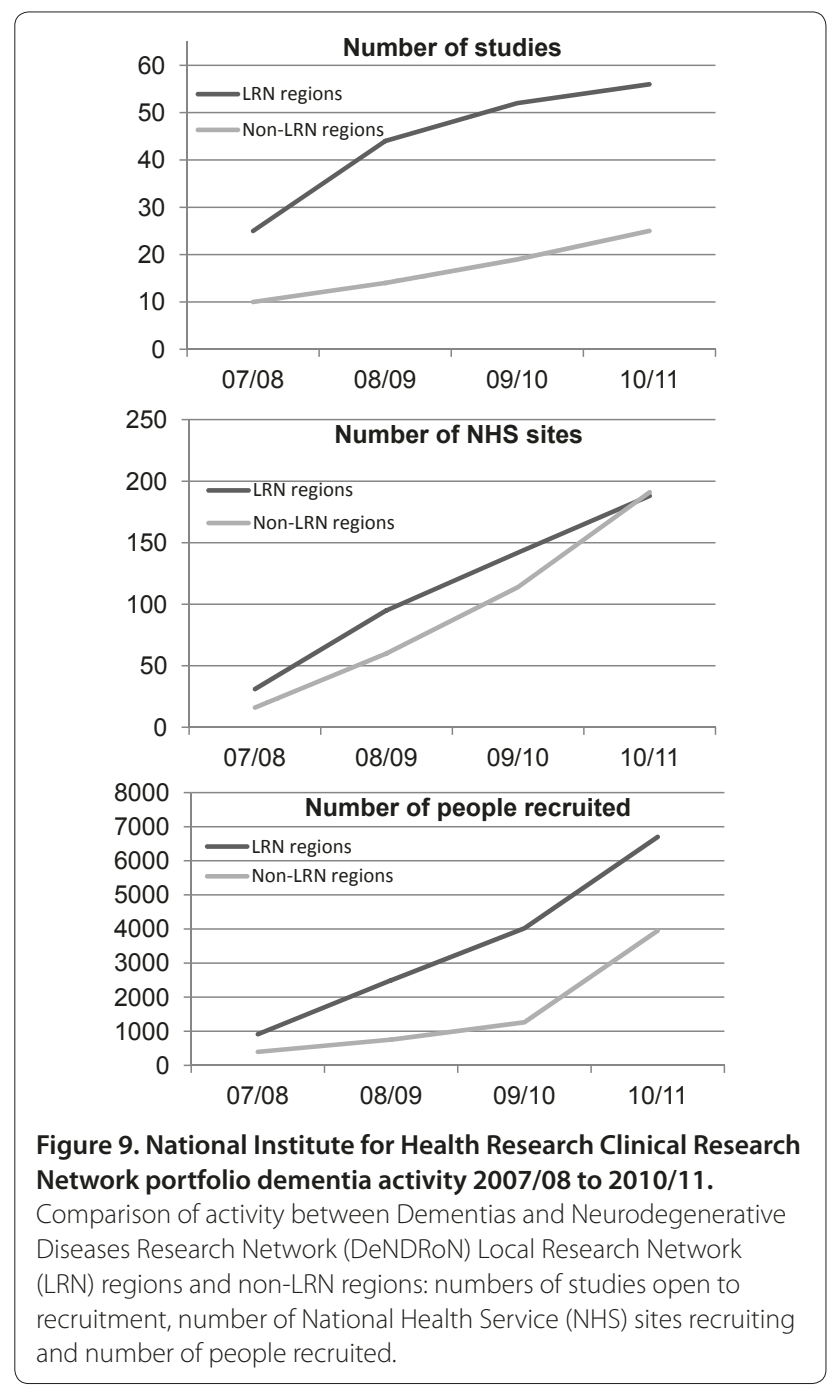

\section{Impact on patient outcomes}

At present, as this analysis suggests, much of the impact of DeNDRoN can only be evaluated in terms of the level of research activity and the performance of clinical studies in England. However, the key success measure for the network will be in the impact of this research activity on people with dementia and their carers. Owing to the long timescale between the delivery of research and the evaluation of the impact of the implementation of research findings, it will be some years before the impact of DeNDRoN on patient outcomes and experience will be known. Indications from the early studies supported by DeNDRoN are encouraging [16-19], however, and there are several areas that might usefully be evaluated in the shorter term.

One such area is the impact on patient experience of being involved in research; and the impact of research activity on the quality of care. Evidence from cancer suggests that participation in clinical trials may benefit an individual over another receiving equivalent treatment outside a trial [20] and that research-active healthcare systems deliver better outcomes for patients than research-inactive systems [21]. DeNDRoN has an impact on levels of research activity in NHS providers, so it would be valuable to understand the impact of that activity on patient outcomes.

A second area is the impact of DeNDRoN's patient and public involvement activities on patient awareness and involvement in the research process, which have been suggested to be major factors in increasing recruitment to clinical trials [22].

Another area for evaluation is the extent to which research is becoming part of the culture of dementia services in England, and the role of DeNDRoN in supporting cultural change.

A final area for evaluation is the development of working relationships between researchers and clinicians from different backgrounds, specialties and interests; and their impact on dementia research.

\section{Conclusions}

There has been a fundamental change in the way clinical research is supported in England over the past 5 years. During this period a new network to support clinical research in dementias and neurodegenerative diseases has been established. As a result there is a significantly larger and more experienced workforce in England to support the delivery of dementia studies. The workforce is more embedded in NHS clinical services than before the networks and has increased the capacity of the NHS in England to deliver dementia research. There is increasing collaboration across the dementia research community and a growing number of clinical services are involved in research. There is also more interaction between old-age psychiatrists, geriatricians and neurologists, and between the different NHS trusts in which these specialists work.

The network is increasing the number of dementia studies developed in England and the capacity of the NHS to deliver them. This is increasing access to research for patients and improving the performance of studies. The development of dedicated infrastructure, systems and processes to support dementia and neurodegenerative diseases research in England has already had a significant impact and provides a platform for future developments. With the prioritisation of dementia research by the UK Government - and in particular the recent investment in dementia translational research facilities, including the establishment of a dementia translational research collaboration - there is an exciting opportunity for the dementia research community in England to collaborate towards answering further key research questions more quickly. 

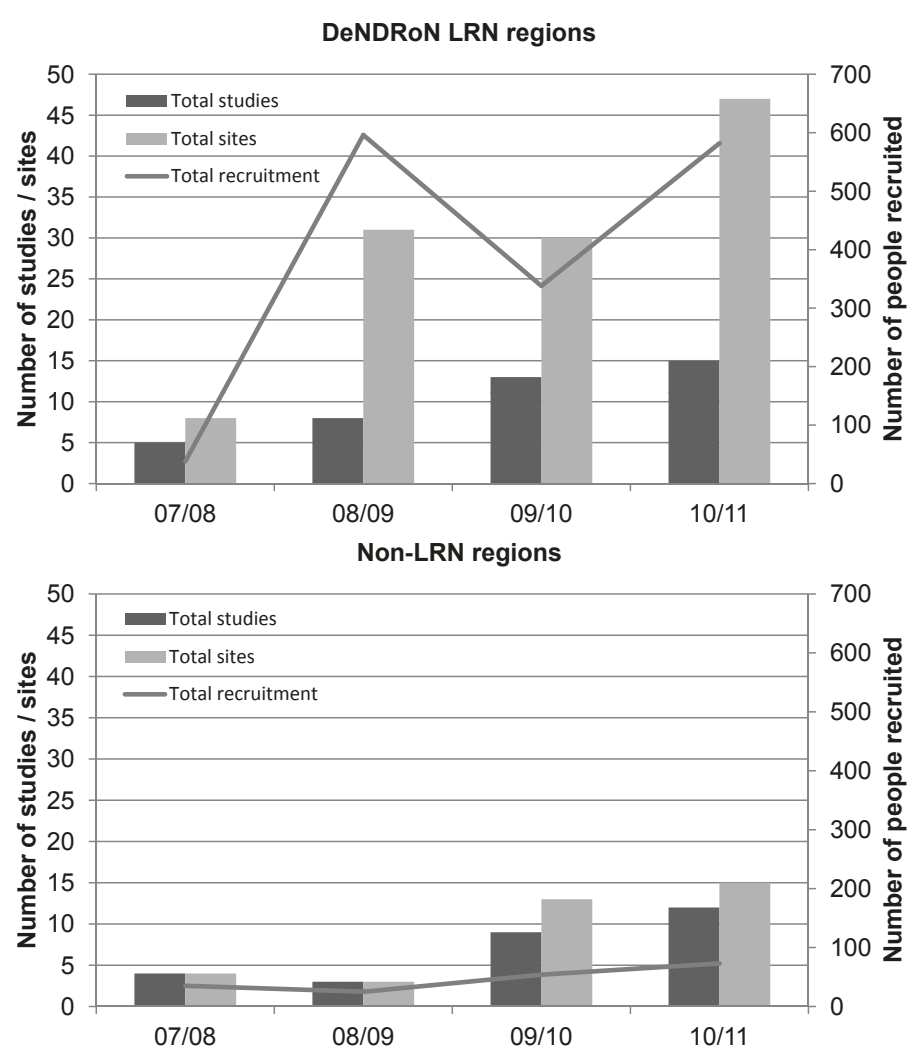

Figure 10. National Institute for Health Research Clinical Research Network portfolio commercial dementia activity 2007/08 to 2010/11. Comparison of activity between Dementias and Neurodegenerative Diseases Research Network (DeNDRoN) Local Research Network (LRN) regions and non-LRN regions: numbers of studies open to recruitment, number of National Health Service sites recruiting and number of people recruited.

DeNDRoN should consider extending its role in leadership and coordination of research development to support the community in responding to this opportunity, focusing on pulling through research from translational medicine to implementation into clinical practice. DeNDRoN also offers the international research community and commercial research sponsors better access to researchers and patients in England to support international efforts to alleviate the suffering caused by dementia and neurodegenerative diseases.

\section{Abbreviations}

CLRN, Comprehensive Local Research Network; DemReg, North Thames Dementia Register; DeNDRoN, Dementias and Neurodegenerative Diseases Research Network; INTERACT, Integrating Networks and Embedding Research in Clinical Services Project; LRN, Local Research Network; MHRN, Mental Health Research Network; NHS, National Health Service; NIHR, National Institute for Health Research; R\&D, research and development; UCL, University College London.

\section{Competing interests}

The authors declare they are officers of DeNDRoN and receive funding (via their employing universities) from the Department of Health for their DeNDRoN roles.

\section{Acknowledgements}

DeNDRoN is funded by the Department of Health in England via the NIHR.

\section{Author details}

'Department of Neurodegenerative Diseases, Institute of Neurology, University College London, London WC1N 3BG, UK. ${ }^{2}$ Newcastle University, Wolfson Research Center, Institute of Ageing \& Health, Newcastle Upon Tyne NE4 5PL, UK.

Published: 6 July 2012

\section{References}

1. Best Research for Best Health [http://www.dh.gov.uk/en/ Publicationsandstatistics/Publications/PublicationsPolicyAndGuidance/ DH_4127127]

2. Eligibility Criteria for NIHR Clinical Research Network Support [http://www. crncc.nihr.ac.uk/Resources/NIHR\%20CRN\%20CC/Portfolio/Documents/ Eligibility\%20Criteria\%20for\%20NIHR\%20Clinical\%20Research\%20 Network\%20Support\%20-\%20Version\%204\%20(February\%202011).pdf]

3. Luengo-Fernandez R, Leal J, Gray A: Dementia 2010: The Economic Burden of Dementia and Associated Research Funding in the United Kingdom. Cambridge: Alzheimer's Research Trust; 2010.

4. Luengo-Fernandez R, Leal J, Gray A: UK research expenditure on dementia, heart disease, stroke and cancer: are levels of spending related to disease burden? Eur J Neurol 2012, 19:149-154.

5. Alzheimer's Research UK: Defeating Dementia 2012. Building Capacity to Capitalise on the UK's Research Strengths. Cambridge: Alzheimer's Research UK; 2012.

6. NHS Staff Turnover Statistics 2007-2008 [http://www.ic.nhs.uk/statisticsand-data-collections/workforce/nhs-turnover/ nhs-staff-turnover-statistics-2007-2008]

7. The Ministerial Advisory Group on Dementia Research - Headline Report [http://www.dh.gov.uk/en/Publicationsandstatistics/Publications/ 
PublicationsPolicyAndGuidance/DH_127750]

8. Rossor M: Putting minds to the matter. Public Service Review: UKScience and Technology 2011, 4. [http://www.publicservice.co.uk/article. asp?publication=UK\%20Science $\% 20$ and $\% 20$

Technology\&id=546\&content_name=Health\&article=18478]

9. Department of Health: Prime Minister's Challenge on Dementia. Delivering Major Improvements in Care and Research by 2015. London: Department of Health; 2012. [http://www.dh.gov.uk/en/Publicationsandstatistics/ Publications/PublicationsPolicyAndGuidance/DH_133170]

10. Campbell MK, Snowdon C, Francis D, Elbourne D, McDonald AM, Knight R, Entwistle V, Garcia J, Roberts I, Grant A: Recruitment to randomised trials: strategies for trial enrollment and participation study. The STEPS study. Health Technol Assess 2007, 11:ix-105.

11. Rick CE, Clarke CE, Dowling FP, Gray R, Ives NJ, Patel S, Wheatley K, Winkles NP: Local Research Networks are an effective way of improving recruitment to clinical trials [abstract 632]. In Proceedings of the Movement Disorder Society's $12^{\text {th }}$ International Congress: June 22-26 2008; Chicago, IL. Milwaukee: Movement Disorder Society International; 2008. [http://www. movementdisorders.org/congress/congress08/2008final.pdf]

12. Rick CE, Clarke CE, Wheatley K, Winkles NP, Patel S, Ives NJ, Gray R: Reasons to be optimistic about the NIHR UK Clinical Research Collaboration - impact of DeNDRoN clinical research network on recruitment to PD MED trial. BM 2010, 340:c2375. [http://www.bmj.com/rapid-response/2011/11/02/ reasons-be-optimistic-about-nihr-uk-clinical-research-collaboration-impact

13. Iliffe S, Pashoja AC, Lowery DP, Warner J: EVIDEM-E: Challenges of Recruitment to a Trial of Therapeutic Intervention in Dementia. International Association for Geriatrics \& Gerontology 7th European Congress, Bologna, 16 April 2011 [oral presentation].

14. Lowery DP, Pashoja AC, Warner J, lliffe, S: Clinicians as recruiters to dementia trials: lessons learned from EVIDEM-E project. Int J Geriatric Psychiatry 2011, 26:765-769.

15. Cerga-Pashoja A, Lowery D, Bhattacharya R, Griffin M, Iliffe S, Lee J, Leonard C, Ricketts S, Strother L, Waters F, Ritchie CW, Warner J: Evaluation of exercise on individuals with dementia and their carers: a randomised controlled trial. Trials 2010, 11:53.

16. Banerjee S, Hellier J, Dewey M, Romeo R, Ballard C, Baldwin R, Bentham P, Fox C, Holmes C, Katona C, Knapp M, Lawton C, Lindesay J, Livingston G, McCrae
N, Moniz-Cook E, Murray J, Nurock S, Orrell M, O'Brien J, Poppe M, Thomas A, Walwyn R, Wilson K, Burns A: Sertraline or mirtazapine for depression in dementia (HTA-SADD): a randomised, multicentre, double-blind, placebocontrolled trial. The Lancet 2011, 378:403-411.

17. Shatunov A, Mok K, Newhouse S, Weale ME, Smith B, Vance C, Johnson L, Veldink JH, van Es MA, van den Berg LH, Robberecht W, Van Damme P, Hardiman O, Farmer AE, Lewis CM, Butler AW, Abel O, Andersen PM, Fogh I, Silani V, Chiò A, Traynor BJ, Melki J, Meininger V, Landers JE, McGuffin P, Glass JD, Pall H, Leigh PN, Hardy J, et al:: Chromosome 9p21 in sporadic amyotrophic lateral sclerosis in the UK and seven other countries: a genome-wide association study. Lancet Neurol 2010, 9:986-994.

18. Gray R, Patel S, Ives N, Rick C, Jenkinson C, Wheatley K, Williams A, Clarke CE: A large randomised trial assessing quality of life in patients with early PD: results from PD MED Early [abstract]. In Parkinsonism \& Related Disorders, Volume 18, Supplement 2, pp. i-iv, S1-S250 (January 2012), p. S32. ISSN 13538020, doi:10.1016/S1353-8020(11)70205-X

19. Howard R, McShane R, Lindesay J, Ritchie C, Baldwin A, Barber R, Burns A, Dening T, Findlay D, Holmes C, Hughes A, Jacoby R, Jones R, Jones R, McKeith I, Macharouthu J, O'Brien J, Passmore P, Sheehan B, Juszczak E, Katona C, Hills R, Knapp M, Ballard C, Brown R, Baneriee S, Onions C, Griffin M, Adams J, Gray $R$, et al:: Donepezil and memantine for moderate-to-severe Alzheimer's disease. NEngl J Med 2012, 366:893-903.

20. Braunholtz DA, Edwards SJL, Lilford RJ: Are randonized clinical trials good for us (in the short term)? Evidence for a 'trial effect'. J Clin Epidemio/ 2001, 54:217-224.

21. Selby $P$ : The impact of the process of clinical research on health service outcomes. Annals Oncol 2011, 22(Suppl 7):vii2-vii4.

22. Caldwell P, Hamilton S, Tan A, Craig C: Strategies for increasing recruitment to randomised controlled trials. PLoS Med 2010, 7. [http://www. plosmedicine.org/article/info\%3Adoi\%2F10.1371\%2Fjournal.pmed.1000368]

doi:10.1186/alzrt126

Cite this article as: Kotting $\mathrm{P}$, et al.: Supporting clinical research in the NHS in England: the National Institute for Health Research Dementias and Neurodegenerative Diseases Research Network. Alzheimer's Research \& Therapy 2012, 4:23. 